\title{
Modelos de gestão pública da Secretaria de Saúde de Pernambuco: implicações na gestão do trabalho
}

\author{
Public management models of Pernambuco Health Department: \\ implications for labor management
}

Vanessa Gabrielle Diniz Santana', Pedro Miguel dos Santos Neto ${ }^{2}$

RESUMO Trata-se de um estudo de caso único com enfoque incorporado de base qualitativa, exploratório e retrospectivo, cujo objetivo foi analisar como a gestão do trabalho em saúde é praticada em diferentes modelos de gestão pública no estado de Pernambuco, estudando dois hospitais: um gerenciado por Organização Social de Saúde e o outro sob gestão direta. Foram realizadas entrevistas semiestruturadas, além de análise documental no período de 2007 a 2013. O estudo apontou que o modelo de gestão pública se deu a partir da necessidade de expansão da rede de saúde; além de que existe uma Política de Gestão do Trabalho implantada na Secretaria Estadual de Saúde, com disparidade nos hospitais estudados.

PALAVRAS-CHAVE Organização social. Recursos humanos. Gestão em saúde.

\begin{abstract}
This is a single case study with a built-in focus of qualitative basis, exploratory and retrospective, whose aim was to analyze how the health work management is practiced in different models of public management in the State of Pernambuco, studying two hospitals: one managed by Social Health Organization and the other under direct management. Semi-structured interviews were conducted, as well as document analysis for the period 2007 to 2013. The study pointed that the public management model was based on the need of the health network expansion; besides that, there is a Labor Management Policy implemented in the State Department of Health, with disparity in the hospitals studied.
\end{abstract}

KEYWORDS Social organization. Human resources. Health management.

1Fundação Oswaldo Cruz (Fiocruz), Centro de Pesquisas Aggeu Magalhães - Recife (PE), Brasil.

vgds@hotmail.com

2 Fundação Oswaldo Cruz (Fiocruz), Centro de Pesquisas Aggeu Magalhães - Recife (PE),

Brasil.

pedromiguel@cpqam.

fiocruz.br 


\section{Introdução}

A gestão do trabalho em saúde é uma questão que tem merecido relevância em todas as instituições que buscam a correta adequação entre as necessidades da população usuária e os seus objetivos institucionais (ARIAS, 2006). Pensar em gestão do trabalho, como eixo da estrutura organizacional dos serviços de saúde, significa pensar estrategicamente, uma vez que a produtividade e a qualidade dos serviços oferecidos à sociedade serão, em boa parte, reflexos da forma e das condições com que são tratados os que atuam profissionalmente na organização (MACHADO, 2000).

Conforme expresso na Norma Operacional Básica de Recursos Humanos do Sistema Único de Saúde (NOB/RH-SUS), a área de gestão do trabalho em saúde passou a ser considerada como uma das questões mais complexas do Sistema Único de Saúde (SUS). A norma enfatiza a centralidade do trabalho, afirmando a necessidade da valorização profissional e da regulação das relações de trabalho para o fortalecimento dos ideais da Reforma Sanitária, e identifica a necessidade de um resgate da gestão do trabalho em saúde como política pública; além disso, retoma a expectativa de que os trabalhadores atuem como agentes de mudança da prática no setor público (BRASIL, 2002).

A gestão do trabalho em saúde é um tema central, pois, a força de trabalho desempenha um papel fundamental na produção e utilização dos serviços de saúde. Trata-se também de um tema complexo por não ser uma ciência exata e pelos resultados serem dependentes de um grande número de fatores. O sucesso das ações de saúde depende, portanto, da organização do trabalho, ou seja, de definições sobre a quantidade, competências, distribuição, treinamento e condições de trabalho dos profissionais da saúde (DUSSAULT; SOUZA, 1999).

O mundo contemporâneo vem experimentando inovações nos processos de gestão, horizontalizando as funções de gerência, (re)omeando e (res)significando a gerência de recursos humanos para a gestão de pessoas no sentido de humanizar a área e valorizar o capital humano e, ao mesmo tempo, buscar qualidade, produtividade e competitividade. Ou seja, desenvolver novos estilos gerenciais, visto que as formas tradicionais já não respondem às exigências de competitividade no mercado. Ao trabalhador, são imputados novos atributos via ampliação de seus conhecimentos e busca pela polivalência funcional, para garantir espaços no mundo cada vez mais restrito de oportunidades de emprego (PIERANTONI ET AL., 2008).

Este estudo foi realizado na Secretaria Estadual de Saúde do Estado de Pernambuco (SES/PE) que conta com um quadro de 25.211 profissionais de saúde, contém 185 Municípios, tem uma área de 98.311.616 Km2 e possui uma população de 8.796 .032 habitantes (PERNAMBUCO, 2014B).

O estado de Pernambuco é dividido em 4 macrorregiões de saúde; 12 Gerências Regionais de Saúde (Geres); 23 hospitais da rede própria, 9 hospitais gerenciados por Organizações Sociais de Saúde (OSS), sendo 6 que foram passados de gestão direta para gerenciamento por OSS e 4 novos hospitais metropolitanos inaugurados já com esse tipo de gerenciamento a partir de 2010; além de 14 Unidades de Pronto Atendimento (Upas) e 9 Unidades Pernambucana de Atenção Especializada (Upae) (PERNAMBUCO, 2013A).

A partir de 2006, com a gestão do governador Eduardo Campos, veio a proposta de construção de hospitais que fossem gerenciados por OSS. Para a construção e a aquisição dos equipamentos das unidades gerenciadas pelas OSS, foi utilizado recurso do governo estadual. Nesses casos os profissionais de saúde também são contratados pelas OSS conforme a Consolidação das Leis Trabalhistas (CLT).

Salienta-se que a Política de Gestão do Trabalho e Educação em Saúde na SES/PE existe apenas para as unidades sob gestão direta e, nas unidades sob gerenciamento 
das OSS, é de competência da organização instituí-la ou não. O objetivo deste artigo é analisar como a gestão do trabalho em saúde é praticada nos diferentes modelos de gestão pública em dois hospitais que estão sob a responsabilidade da SES/PE.

\section{Métodos}

Trata-se de um estudo de caso único com enfoque incorporado de base qualitativa, exploratório e retrospectivo. Para o desenvolvimento da análise proposta, foram coletados dados referentes ao período de 2007 a 2013. A opção por esse período coincidiu com a implantação do novo modelo de gestão no estado de Pernambuco e com o início do governo de Eduardo Campos.

De acordo com Yin (2001), estudo de caso único é aplicado para testar uma teoria bem formulada, seja para confirmá-la, seja para contestá-la, seja ainda para entender a teoria. Nesse caso, o estudo deve satisfazer todas as condições para testar a teoria. Outra aplicação desse estudo é quando o caso único se mostra revelador, quando o pesquisador tem a chance de observar um fenômeno anteriormente inacessível à investigação científica. Outra aplicabilidade do estudo é quando ele é utilizado como introdução a um estudo mais apurado ou, ainda, como caso-piloto para a investigação. Dessa forma, as três aplicações em questão, o estudo de caso único foi aplicado neste estudo, já que serviu para testar a teoria de que há diferenciação entre os modelos de gestão implantadas nas unidades de saúde sob gestão direta e nas unidades sob gestão OSS, além de que serviu também para entender como está sendo praticada a gestão do trabalho em saúde no estado de Pernambuco. Este estudo é revelador já que não existia um estudo sobre as OSS e gestão do trabalho no referido estado.

Ainda de acordo com Martins (2008), o estudo de caso incorporado é aquele no qual a situação é avaliada a partir de diferentes unidades ou níveis de análise, que podem ser setores diferentes de uma determinada instituição (setor de vendas e setor de produção, por exemplo), podem ser atividades (processo de planejamento e processo de implantação, por exemplo). Cada um dos níveis de análise ou unidades pode ser feito com critérios distintos. Neste estudo, foram analisadas duas unidades hospitalares a partir dos seguintes critérios de análise: contexto, processo, conteúdo e atores.

Segundo Minayo e Sanches (1993), a abordagem qualitativa expressa a fala cotidiana, seja nas relações afetivas e técnicas, seja nos discursos intelectuais, burocráticos e políticos. Aprofunda-se no mundo dos significados das ações e relações humanas, um lado não perceptível e não captável em equações, médias e estatísticas (MINAYO, 2002).

Com o intuito de contemplar as categorias de análise e suas variáveis, para obtenção dos objetivos do estudo, foi necessário lançar mão de duas formas de análise de dados: análise documental e análise do conteúdo narrativo das entrevistas.

A análise documental buscou identificar nas fontes utilizadas informações relevantes para a compreensão da análise da política de gestão do trabalho em saúde, além de entender como os diferentes modelos de gestão surgiram e estão postos na SES/PE.

Para a análise do conteúdo das entrevistas semiestruturadas, que foram aplicadas aos informantes-chave, utilizou-se o método de análise de políticas de Walt e Gilson (1994) em que as categorias de análise estudadas são: contexto (político, econômico, social e serviços de saúde), conteúdo (programas, projetos, propostas e objetivos), processo (entrada na agenda, formulação, implantação e avaliação) e atores (individuais e institucionais).

A partir das entrevistas e dos documentos analisados, foram identificados nos dois hospitais estudados os diferentes modelos de gestão pública e como estes modelos implicam a gestão do trabalho em saúde considerando as fases desenvolvidas no dia a dia 
organizacional, verificando como o recrutamento e seleção, a aplicação/lotação, o desenvolvimento e a avaliação são impactados pelos diferentes modelos administrativos adotados pela SES/PE.

Foram utilizados os quatro elementos de análise. No 'contexto', foram identificadas a Política de Saúde e Política de Gestão do Trabalho, a legislação vigente e as unidades de saúde com seus diferentes modelos de gestão; no 'processo', foram identificados como o modelo de gestão foi viabilizado, como os profissionais foram lotados nas unidades de saúde, além das formas de desenvolvimento dentro das unidades de saúde, a partir do Ciclo utilizado por Malik; no 'conteúdo', os contratos de gestão, além de o que e como a SES/PE cobra dos entes contratados para gerir as unidades de saúde; nos 'atores', foram identificados os implementadores da Política de Gestão do Trabalho na SES/PE e os trabalhadores de saúde das duas unidades pesquisadas. Em relação aos atores, neste artigo não serão abordados os resultados encontrados.

No Processo da Política, foram utilizadas como indicador as fases do desenvolvimento do dia a dia organizacional proposto por Malik (1998), observando o Suprimento/recrutamento, Desenvolvimento e Avaliação. No suprimento, foram descritas todas as formas de inserção nas unidades, formas de contratação entrada e saídas dos profissionais; no Desenvolvimento, destacaram-se as iniciativas de capacitação, formação, educação em saúde realizada pelas unidades; na Avaliação, foi estudado como é realizada a Avaliação de Desempenho (AD) dos profissionais assim como os incentivos ofertados.

Foram realizadas seis entrevistas semiestruturadas individuais com gestores das unidades pesquisadas, sendo suficientes para a análise qualitativa do estudo. Foi entrevistado o secretário executivo de gestão do trabalho e educação na saúde, diretores das duas unidades estudadas além de outro gestor que foi escolhido pelo diretor da Unidade. No
Hospital Otávio de Freitas (HOF), foi escolhido o gestor de enfermagem, e no Hospital Metropolitano Norte Miguel Arraes de Alencar (HMNMAA), o diretor médico. O secretário executivo de atenção à saúde estava previsto para ser entrevistado, porém ele solicitou que a assessora técnica respondesse à entrevista. A escolha pelos profissionais em questão foi definida com vistas a compreender a Política de Gestão do Trabalho nos diferentes modelos de gestão.

É importante destacar que, como as entrevistas foram semiestruturadas, foi possível uma adaptação das perguntas conforme a evolução das entrevistas. Ressalta-se que apenas uma pessoa entrevistou todos os gestores.

Também foi realizada a pesquisa documental, analisando documentos sobre a política estadual, análise de políticas, modelos de gestão, gestão do trabalho em saúde. Para Godoy (1995), a pesquisa documental é aquela realizada com base em documentos ou com pessoas, mediante registros, atas, circulares, jornais, revistas, ofícios, entre outros tipos de documentos. Nessa modalidade de pesquisa, é feito o exame e tratamento analítico desses documentos escritos.

Este trabalho foi desenvolvido obedecendo aos preceitos éticos definidos na Resolução do Conselho Nacional de Saúde $n^{\circ} 466$ de 12 de dezembro de 2012 e foi submetido, e aprovado, pelo Comitê de Ética em Pesquisa (CEP/CPQAM), sob o parecer $n^{\circ}$ 679.351. A SES/PE forneceu Carta de Anuência para a realização do estudo.

\section{Resultados e discussão}

A Reforma Gerencial no Brasil foi introduzida em 1995, quando foi criado o Ministério da Administração e Reforma do Estado (Mare). Seu titular, Luiz Carlos Bresser Pereira, estava afinado com o pensamento internacional na área de administração pública. Aliado político de Fernando 
Henrique Cardoso de longa data, Bresser teve plena liberdade para montar sua equipe ministerial, o que lhe permitiu a formulação de uma proposta inovadora cujos temas da agenda política incluíram: a) ajuste fiscal, com redução do gasto público; b) reformas econômicas orientadas para o mercado, com ênfase na privatização de empresas estatais; c) reforma da previdência social; d) reforma do aparelho do Estado e maior capacidade de governo ou governança (BRESSSER-PEREIRA; SPINK, 1998).

Em Pernambuco, a primeira lei que instituiu as OSS e também as Organizações da Sociedade Civil de Interesse Público (Oscips) foi publicada em 2000, no governo de Jarbas Vasconcelos, sendo aprovada pela Assembleia Legislativa. O modelo de gerenciamento via OSS adotado pela SES iniciou apenas em 2010, com a inauguração do HMNMAA. Em 2013, foi publicada a Lei ${ }^{\circ}$ 15.210 que dispõe sobre as OSS no âmbito do estado de Pernambuco e que rege todo o funcionamento e monitoramento dos contratos de gestão celebrados entre a SES e a OSS (PERNAMBUCO, 2013C).

As Organizações Sociais (OS) foram criadas por meio da Medida Provisória ${ }^{0}$ 1.591, de 9 de outubro de 1997, reeditada sete vezes, convertida na Lei no 9.637, de 15 de maio de 1998, que dispõe sobre a qualificação de entidades como organizações sociais. Poderão ser qualificadas como OS, pessoas jurídicas de direito privado, sem fins lucrativos, cujas atividades sejam dirigidas ao ensino, à pesquisa científica, ao desenvolvimento tecnológico, à proteção e preservação do meio ambiente, à cultura e à saúde (BRASIL, 1997).

$\mathrm{Na}$ condição de entidades de direito privado, as OS tenderão a assimilar características de gestão cada vez mais próximas das praticadas no setor privado, o que representa, entre outras características: a contratação de pessoal nas condições de mercado; a adoção de normas próprias para compras e contratos; e ampla flexibilidade na execução do seu orçamento (BRASIL, 1997).
A SES/PE adotou o modelo de gestão com o gerenciamento das unidades hospitalares por OSS a partir de vários fatores, entre eles, os que mais se destacaram nas entrevistas foram: decisão política, necessidade de contratação de pessoal para além da Lei de Responsabilidade Fiscal, superlotação dos hospitais em 2006 e necessidade de expandir a rede de saúde com contratação de pessoal de forma imediata.

Conforme Correia (2008), a administração dos serviços públicos de saúde passou a se pautar por medidas de flexibilização, visando maximizar a relação custo/benefício, resultando na privatização e na terceirização dos serviços de saúde, e também no repasse de serviços e recursos públicos para OS, Oscips, fundações de apoio e cooperativas de profissionais de medicina. $\mathrm{O}$ resultado foi a ampliação da oferta de serviços de saúde no setor privado.

Ressalta-se que, de fato, há a dificuldade de contratação de pessoal devido à Lei de Responsabilidade Fiscal, Lei Complementar $\mathrm{n}^{\mathrm{o}} 101$, de 4 de maio 2000, que prevê que a despesa total com pessoal, em cada período de apuração no Estado, não poderá exceder $60 \%$ da receita corrente líquida (BRASIL, 2000). Foi observado a partir das entrevistas que a OSS veio como um modelo que atendia a essa necessidade de expansão da rede, sem expandir gasto com folha de pessoal do tesouro; ela respondia a uma possibilidade de flexibilizar a contratação.

O primeiro contrato de gestão foi celebrado em 25 de novembro de 2009, entre a SES e a Fundação Professor Martiniano Fernandes - Imip Hospitalar, para operacionalizar a gestão hospitalar e executar ações e serviços de saúde no HMNMAA.

Campos (2007) define o contrato de gestão como uma modalidade de relação interinstitucional com grande potencialidade. Primeiro, porque explicita os programas concretos de cada gestor; segundo, introduz em alguma medida uma modalidade real de cogestão sem diminuir a autonomia e a 
responsabilidade do encarregado pela execução da atenção à saúde; terceiro, define com clareza a responsabilidade sanitária de cada ente federado; e, ainda, institui um sistema regular de avaliação de resultados bastante vinculado à dinâmica da própria gestão. $\mathrm{O}$ autor ainda cita que o contrato pode ser utilizado tanto entre entes federados como também entre o gestor local e o prestador de serviços.

Ressalta-se que o imóvel e os bens móveis são pertencentes à SES e que compete à OSS assegurar a organização, administração e gerenciamento do Hospital, o provimento dos insumos (materiais) e medicamentos e a garantia do quadro de recursos humanos qualificados e compatíveis com o porte da unidade e serviços contratualizados, podendo o estado intervir na administração da unidade hospitalar, caso haja risco quanto à continuidade dos serviços de saúde.

Na Lei $n^{0} 15.210 / 2013$, foi instituído que o prazo de vigência do contrato de gestão pode ser estendido em até dez anos, desde que restem demonstradas as vantagens da medida e o pleno atendimento das metas pactuadas, conforme parecer elaborado pela Comissão de Avaliação e aprovado pela autoridade máxima do órgão supervisor do contrato de gestão (PERNAMBUCO, 2013C).

Em relação aos servidores públicos efetivos estaduais, eles poderão ser cedidos às OSS, sendo mantido o seu vínculo com o estado, nos termos do estatuto dos servidores (Lei $n^{\circ}$ 6.123, de 20 de julho de 1968), computando-se o tempo de serviço prestado para todos os efeitos legais, inclusive promoção por antiguidade e aposentadoria, está vinculada ao desconto previdenciário próprio dos servidores públicos do estado. A Lei ainda abrange que o servidor poderá, a qualquer tempo, mediante requerimento ou por manifestação da OSS, ter sua cessão cancelada. Além de que o servidor público cedido poderá receber da OSS estímulo remuneratório por resultados, por meio de recursos próprios da entidade, que não serão incorporados à remuneração (PERNAMBUCO, 1968; PERNAMBUCO, 2013C).

Na SES/PE, existem oito OSS contratualizadas: Imip Hospitalar, Santa Casa de Misericórdia, Hospital Maria Lucinda, Hospital do Tricentenário, Fundação Altino Ventura, Hospital do Câncer, Associação de Proteção à Maternidade e à Infância de Surubim (Apami), Instituto Pernambucano de Assistência à Saúde (Ipas). Essas OSS administram 9 hospitais, 14 Upas, 9 Upae, com um total de recursos investidos, com contratos de gestão, no valor de R $\$ 713.643 .307,44$ ao ano em 2014 (PERNAMBUCO, 2014A).

De acordo com Campos (2007), esse novo desenho para a administração direta deveria resolver alguns dos entraves já identificados e decorrentes da atual legislação, que simplesmente estendeu para o SUS o modelo de gestão do Estado brasileiro, sem considerar as especificidades do campo da saúde e do SUS em particular. Assim, esses hospitais e organizações do SUS poderiam incorporar, entre outras, as seguintes características: maior autonomia e integração ao sistema, mediante contratos de gestão; financiamento misto (parte fixa e outra variável); conselho gestor; direção executiva composta mediante critérios técnicos e seleção pública, com mandato, e não como cargos de confiança; nova política de pessoal com a possibilidade de alguma modalidade de contratação do tipo emprego público, com carreiras e $\mathrm{AD}$ (remuneração mista); políticas de recrutamento e de educação continuada estadual e nacional.

Foram estudados dois Hospitais da SES; o HOF e o HMNMAA, pertencentes ao estado de Pernambuco. O HOF sob o gerenciamento da SES, e o HMNMAA tem a sua administração gerencial centrada na OSS.

Ambos os hospitais estudados são de alta complexidade e possuem emergências que devem ser referenciadas pelas Upas, Central de Regulação e Serviço de Atendimento Móvel de Urgência (Samu). O HOF pertence à gestão estadual direta, possui 652 leitos, 1.695 profissionais, sendo referência em 
Urologia e no tratamento de doenças respiratórias, em especial a tuberculose (PERNAMBUCO, 2013B). O HMNMAA é um grande hospital de trauma e foi o primeiro, na rede pública de saúde, a adotar o modelo de gestão via OSS, possui 180 leitos, 977 profissionais, e é referência estadual em endoscopia digestiva e traumato-ortopedia (PERNAMBUCO, 2013B).

O modelo atualmente vigente na administração direta para a gestão de hospitais e serviços especializados se caracteriza por rigidez na execução orçamentária, emperramento na administração de pessoal, excessiva interferência político-partidária. Tudo isso tem levado grande número de serviços públicos à burocratização e mesmo à degradação organizacional. Ao longo dos anos, gestores inventaram estratégias para contornar parte dessas dificuldades, uma delas é delegar a gestão dos hospitais a entidades civis privadas, criando-se leis e normas que permitiram a existência de OSS ou Oscip integradas à rede do SUS. Esta última linha de mudança indica uma desistência da administração direta, já que investe em modalidades de gestão com base em contratos entre o gestor - restrito ao papel de regulador - e entes privados sem fim lucrativos (CAMPOS, 2007).

Alguns resultados díspares encontrados nos dois hospitais, no que se refere à gestão do trabalho em saúde, estão descritos logo abaixo.

Em relação à 'contratação/formas de inserção' dos profissionais nos serviços de saúde, destacam-se no HOF: concurso público, seleção pública simplificada, cooperativas de anestesiologia e terceirizados. Ainda existe outra forma que não é computada como entrada no serviço, e sim como forma de reposição imediata/rápida de servidores que é o plantão extraordinário.

O concurso público é amparado pela Constituição Federal de 1988 em seu Artigo 37 e também pelo Estatuto dos Funcionários Públicos do Estado de Pernambuco, Lei ${ }^{\circ}$ 6.123, de 20 de julho de 1968. Os últimos concursos públicos da SES aconteceram, em
2013, apenas para médicos e, em 2014, para os outros profissionais de saúde (BRASIL, 1988; PERNAMBUCO, 1968).

As seleções públicas simplificadas são regidas pela Lei $\mathrm{n}^{\circ} 14.547$, de 21 de dezembro de 2011. Para tanto, os profissionais de saúde passam por um processo seletivo simplificado de provas e currículo ou apenas análise curricular e, após aprovação, são contratados para atender às necessidades de excepcional interesse público. Esses profissionais são contratados por tempo determinado, podendo as contratações ser prorrogadas por um período de até seis anos (PERNAMBUCO, 2011).

Conforme dados da SES, de janeiro de 2011 até dezembro de 2014, foram realizadas 61 seleções públicas simplificadas para diversos cargos de saúde, sendo contratados mais de 5 mil profissionais.

O plantão extraordinário ainda não está amparado em nenhuma legislação, porém existe a necessidade de reposição rápida e emergencial dos profissionais nas unidades de saúde, pela ausência de servidores em plantões críticos. Esse tipo de reposição acontece nas unidades hospitalares, conforme dados das entrevistas realizadas.

Morici e Barbosa (2013), estudando hospitais de Belo Horizonte, relataram que o ingresso nas instituições administradas segundo as regras públicas se dá prioritariamente por meio de concursos públicos. Esse processo seletivo claramente não é capaz de suprir a assistência com profissionais capacitados com a agilidade necessária, uma vez que os concursos não são realizados regularmente e, quando realizados, são processos morosos, compostos de várias etapas. Nas instituições administradas segundo as regras públicas, a falta de provisão de profissionais aprovados em concurso faz com que seja necessária a contratação de trabalhadores por meio de outros vínculos mais flexíveis, garantindo assim o número adequado de profissionais para o atendimento assistencial. Essa contratação contraria as leis vigentes, mas é vista como única alternativa para o 
pleno funcionamento das instituições de saúde pesquisadas. Conclui-se, então, que a tentativa de garantir o ingresso justo de profissionais mediante a realização de concursos públicos, por sua própria ineficiência, gera uma situação paralela de contratação irregular.

Além das formas acima, no HOF, existe empresa terceirizada para a contratação de pessoal, no regime CLT, na área de limpeza, recepção e administração.

Em relação à seleção de profissionais para o HMNMAA, todo o Processo Seletivo é realizado pela OSS que convoca e encaminha para o hospital, contratando por meio do regime CLT.

Foi observado que existe uma alta rotatividade de profissionais no HMNMAA já que existe uma maior facilidade no ato de contratar e demitir. Foi constatado também que existe poder de barganha nos salários ofertados, por exemplo, se paga mais aos profissionais cuja especialidade se encontra escassa no mercado de trabalho.

Morici e Barbosa (2013) corroboram o fato acima ao citar que a remuneração foi diferente nos dois grupos estudados: nos hospitais administrados segundo as regras públicas os salários estão definidos em tabela negociada com o sindicato ou por lei específica, e nos hospitais administrados segundo as regras privadas eles são definidos de acordo com o mercado.

Para o 'desenvolvimento', destacam-se o incentivo às iniciativas de capacitação, promovidas pelas próprias instituições, mediante o aproveitamento de habilidades e de conhecimentos de servidores do quadro de pessoal; o apoio ao servidor público em iniciativas de capacitação, voltadas para o desenvolvimento das competências institucionais e individuais; a garantia de acesso dos servidores a eventos de capacitação interna ou externa ao seu local de trabalho; o incentivo à inclusão das atividades de capacitação como requisito para a promoção do servidor na carreira; e a complementaridade entre o resultado das ações de capacitação e a mensuração do desempenho, as quais constituem diretrizes que reforçam um novo olhar para o desenvolvimento (BRASIL, 2009).

A educação permanente acontece nos hospitais e nas clínicas das unidades sob gestão direta. Existe o eixo do desenvolvimento profissional, o eixo da educação permanente que tanto atua numa perspectiva de educação continuada, voltada para as questões de habilidades e competências de uma determinada demanda, como também para a reorganização do processo de trabalho. Além de que, nas ADs, existem indicadores de incentivo à formação (cursos) e preceptoria que refletem a educação permanente.

Em relação à Política de Educação Permanente na gestão do HMNMAA, o que se pode interpretar das entrevistas é que existem algumas ações de forma incipiente, não sistemática, que fica sob a responsabilidade da OSS, além das atividades dentro da própria unidade, não havendo responsabilização pela SES/PE.

A temática da 'avaliação em saúde' vem sendo alvo de considerações constantes, uma vez que traz implicações em relação ao papel do Estado, tanto quanto formulador e gestor de políticas públicas, quanto na responsabilidade pela geração de informações sólidas apropriadas à abordagem desse complexo objeto. A política de recursos humanos dispensa tratamento diferenciado às ADs, utilizadas até então por grande parte dos gestores como o cumprimento de uma obrigação burocrática desgastante. O que se busca hoje é tornar a $\mathrm{AD}$ um instrumento de gestão, útil para o gestor, o servidor e a sociedade, a quem se destinam os serviços públicos. O papel da gestão de pessoas é ter mecanismos que possibilitem aos servidores atingirem suas metas e que elas se coadunem com as metas da sociedade, objeto de serviços (BRASIL, 2009).

A AD dos servidores da SES está dividida em três macrocomponentes: Avaliação de Desempenho em Estágio Probatório, 
Avaliação de Desempenho para progressão na carreira no Plano de Cargos Carreiras e Vencimentos e Avaliação de Desempenho para percebimento da Gratificação de Desempenho.

Esses três macrocomponentes acontecem dentro do HOF de forma periódica, porém no HMNMAA não há $\mathrm{AD}$ de forma sistemática e com instrumentos próprios.

\section{Conclusões}

Observou-se, no modelo de gestão pública adotado pelo governo do Estado de Pernambuco, a existência de unidades de saúde sendo gerenciadas diretamente pela Administração Pública e de outras por OSS, sendo viabilizado porque existe arcabouço jurídico no SUS, além de ter recursos financeiros instituídos em nível estadual e nacional.

Chama a atenção que a maior motivação da inserção da OSS na SES foi a contratação dos funcionários por meio do regime CLT, pois não contam para o limite fiscal imposto pela Lei de Responsabilidade Fiscal, visto que eles não são empregados diretos do Estado, e sim das OSS contratualizadas.

Em relação à Política de Educação Permanente, esta acontece nas unidades sob gestão direta, porém não está instituída na gestão do HMNMAA, ficando sob a responsabilidade da OSS, sem um direcionamento da SES. Os entrevistados relataram que há uma dificuldade para o desenvolvimento da Política de Educação Permanente por causa da rotatividade dos profissionais.

Diante do exposto, percebe-se que existe uma Política de Gestão do Trabalho implantada na SES, porém com disparidade quando comparados os dois hospitais estudados.

Finaliza-se com algumas recomendações a serem realizadas e incorporadas aos contratos de gestão entre a SES e as OSS: a incorporação de planos de trabalho para os trabalhadores bem definidos com a incorporação de progressões baseadas em tempo de serviço e ADs periódicas; políticas de recrutamento e de educação permanente; avaliações que incorporem a valorização e desenvolvimento dos servidores na instituição inclusive com a diminuição da rotatividade desses funcionários; salários paritários entre as diversas OSS para não haver disputa de mercado; construção de uma agenda de ações de educação permanente e valorização dos trabalhadores das unidades sob gestão OSS no planejamento da Secretaria Executiva de Gestão do Trabalho e Educação em Saúde; entre outras ações que sejam pertinentes para os trabalhadores do SUS.

Por fim, entende-se que o tema estudado não se esgotou, e sim gerou hipóteses para futuras pesquisas. 


\section{Referências}

ARIAS, E. H. L. et al. Gestão do Trabalho no SUS. Cadernos RH Saúde, Brasília, DF, v. 3, n. 1, p. 112-117, mar. 2006.

BRASIL. Constituição (1988). Constituição da República Federativa do Brasil: promulgada em 5 de outubro de 1998. 32. ed. São Paulo: Saraiva, 2003.

Lei Complementar $\mathrm{n}^{\circ}$ 101, de 4 de maio de 2000 Estabelece normas de finanças públicas voltadas para a responsabilidade na gestão fiscal e dá outras providências. Diário Oficial [da] União, Brasília, DF, 4 maio 2000. Disponível em: <http://www.planalto.gov.br/ccivil_03/leis/LCP/Lcp101.htm>. Acesso em: 10 jan. 2017.

Ministério da Saúde. Portaria nº 968, de 11 de dezembro de 2002. Atualizar, na forma dos anexos I, II, III e IV desta Portaria, os Procedimentos de Alta Complexidade e Estratégicos do Sistema de Informações Ambulatoriais e Sistema de Informações Hospitalares - SIA e SIH/SUS. Diário Oficial [da] União, Brasília, DF, 11 dez. 2002. Disponível em: <http://dtr2001.saude.gov.br/sas/PORTARIAS/ PORT2002/PT-968.htm>. Acesso em: 15 mar. 2008.

Ministério da Saúde. O SUS de A a Z: Garantindo saúde nos municípios. 3 ed. Brasília, DF: Ministério da Saúde, 2009. Disponível em: <http://bvsms.saude. gov.br/bvs/publicacoes/sus_az_garantindo_saude_ municipios_3ed_pl.pdf>. Acesso em: 15 nov. 2014

Secretaria da Reforma do Estado Organizações Sociais. Secretaria da Reforma do Estado. Organizações Sociais. 5. ed. Brasília, DF: Ministério da Administração e Reforma do Estado, 1997. (Cadernos MARE da reforma do estado, v. 2).

BRESSER-PEREIRA, L. C.; SPINK, P. (Org.). Reforma do estado e administração pública gerencial. Rio de Janeiro: Fundação Getúlio Vargas, 1998.

CAMPOS, G. W. S. Reforma política e sanitária: a sustentabilidade do SUS em questão? Ciência \& Saúde Coletiva, Rio de Janeiro, v. 12, n. 2, p. 301-306, mar./abr. 2007.
CORREIA, M. V. C. Orientações do Banco Mundial à política de saúde brasileira: flexibilização da gestão e as fundações estatais. In: ENCONTRO NACIONAL DE PESQUISADORES EM SERVIÇO SOCIAL, 11., 2008, São Luís. Anais... São Luís: Associação Brasileira de Pesquisa e Ensino em Serviço Social, 2008. Disponível em: <http://xa.yimg.com/kq/ groups/23089490/692218689/name/Arquivo2.pdf>. Acesso em: 15 dez. 2010.

DUSSAULT, G.; SOUZA, L. E. Gestão de recursos humanos em saúde. Montreal: Universidade de Montreal, 1999. Disponível em: <http://info.worldbank.org/ etools/docs/library/ 206831/Dussault\%20e\%20De\%20 Souza.pdf>. Acesso em: 20 maio 2013.

GODOY, A. S. Pesquisa qualitativa: tipos fundamentais. Revista de Administração de Empresas, São Paulo, v. 35, n. 3, p. 20-29, 1995.

MACHADO, M. H. Gestão do trabalho em saúde no contexto de mudanças. Revista de Administração Pública, Rio de Janeiro, v. 34, n. 4, p. 133-46, jul./ago. 2000.

MALIK, A. M. et al. Gestão de Recursos humanos. São Paulo: Fundação Peirópolis,1998. (Série Saúde e Cidadania, v. 9).

MARTINS, G. A. Estudo de caso: uma reflexão sobre a aplicabilidade em pesquisas no Brasil. Revista de Contabilidade e Organizações, São Paulo, v. 2, n. 2, p. 8-18 jan./abr. 2008.

MINAYO, M. C. S.; SANCHES, O. QuantitativoQualitativo: Oposição ou Complementaridade? Caderno de Saúde Pública, Rio de Janeiro, v. 9, n. 3, p. 239-262, jul./set. 1993.

MINAYO, M. C. S. (Org.). Pesquisa social: teoria, método e criatividade. 21. ed. Petrópolis: Vozes, 2002.

MORICI, M. C.; BARBOSA, A. C. Q. A Gestão de Recursos Humanos em hospitais do Sistema Único de Saúde (SUS) e sua relação ao modelo de assistência: um 
estudo em hospitais de Belo Horizonte, Minas Gerais. Revista de Administração Pública, Rio de Janeiro v. 47, n. 1, p. 205-225, jan./fev. 2013.

PERNAMBUCO. Governo do Estado. Lei no 6.123, de 20 de julho de 1968. Estatuto dos Funcionários Públicos do Estado de Pernambuco. Diário Oficial [do] Estado de Pernambuco, Recife, 13 mar. 1973. Disponível em: $<$ http://www.portaisgoverno.pe.gov.br/c/document_library/get_file?uuid=7c9a503b-c049-4adb-816112676632099 c\&groupId=199085 > . Acesso em: 20 dez. 2014.

Governo do Estado. Lei n ${ }^{\circ}$ 14.547, de 21 de dezembro de 2011. Dispõe sobre a contratação por tempo determinado para atender às necessidades de excepcional interesse público de que trata o inciso VII do art. 97 da Constituição Estadual. Diário Oficial [do] Estado de Pernambuco, Recife, 22 dez. 2011. Disponível em: <http://legis.alepe.pe.gov.br/arquivoTexto. aspx?tiponorma $=1 \&$ numero $=14547 \&$ complemento $=0$ $\&$ ano $=2011 \&$ tipo $=$ TEXTOATUALIZADO $>$. Acesso em: 21 dez. 2014.

Secretaria Estadual de Saúde. Institucional. Disponível em: <http://portal.saude.pe.gov.br/institucional/>. Acesso em: 20 ago. 2013a.

Secretaria Estadual de Saúde. Hospital Metropolitano Norte-Miguel Arraes de Alencar. Disponível em: <http://portal.saude.pe.gov.br/hospitais/hospital-metropolitano-norte-\%E2\%80\%93-miguel-arraes-de-alencar/>. Acesso em: 20 ago. 2013b.
Secretaria Estadual de Saúde. Lei n ${ }^{0} 15.210$, de 19 de dezembro de 2013. Dispõe sobre as Organizações Sociais de Saúde - OSS no âmbito do Estado de Pernambuco. Diário Oficial [do] Estado de Pernambuco, Recife, 20 dez. 2013c. Disponível em: <http://legis. alepe.pe.gov.br/arquivoTexto. aspx?tiponorma=1\&num ero $=15210 \&$ complemento $=0 \&$ ano $=2013 \&$ tipo $=>$. Acesso em: 20 dez. 2014.

Secretaria Estadual de Saúde. Organizações Sociais de Saúde em Pernambuco: mais uma alternativa para consolidação do SUS. Recife, 2014a. Apresentação em Power Point.

Secretaria Estadual de Saúde. Secretaria Executiva de Gestão do Trabalho e Educação na Saúde. Diretoria Geral de Gestão do Trabalho. Recife, jun. 2014b.

PIERANTONI, C. R. et. al. Gestão do trabalho e da educação em saúde: recursos humanos em duas décadas do SUS. Physis, Rio de Janeiro, v. 18, n. 4, p. 685-704, 2008.

WALT, G.; GILSON, L. Reforming the health sector in developing countries: the central role of policy analysis. Health Policy and Plannin, Oxford, v. 9, n. 4, p. 353370, 1994.

YIN, R. K. Estudo de caso: planejamento e métodos. 2. ed. Porto Alegre: Bookman, 2001.

\footnotetext{
Recebido para publicação em agosto de 2016

Versão final em fevereiro de 2017

Conflito de interesses: inexistente

Suporte financeiro: não houve
} 